\title{
Enhancement of Underwater Image using Fuzzy Histogram Equalization
}

\author{
Ghada S. Karam \\ Al-Mustansriah Uni. \\ College of Education \\ Baghdad- Iraq
}

\author{
Ziad M. Abood \\ Al-Mustansriah Uni. \\ College of Education \\ Baghdad- Iraq
}

\author{
Rafal N. Saleh \\ Al-Mustansriah Uni. \\ College of Education \\ Baghdad- Iraq
}

\begin{abstract}
Image enhancement is a process of improving the quality of image by improving its feature. The underwater images suffer from low contrast and resolution due to scattering of light and poor visibility conditions. In this paper we proposed an image enhancement technique to enhance the quality of underwater images. The proposed technique comprises a combination of classical contrast enhancement techniques and fuzzyhistogram equalization techniques. For comparing the performance, some statistics parameter is used. The proposed technique comprises a combination of classical contrast enhancement techniques and fuzzy-histogram equalization techniques. Experiment results demonstrate the effectiveness of fuzzy logic techniques to enhance the quality of the underwater images.
\end{abstract}

\section{Keywords}

Image enhancement, underwater images, fuzzy-histogram equalization.

\section{INTRODUCTION}

Underwater images are essentially characterized by their poor visibility, blur and diminished colors, contrast limited and hazy [1]. In other situation, the image contrast maybe extremely high [2]. The possibilities for long range underwater imaging are quite limited. The light attenuation process is caused by absorption and scattering, which influence the overall performance of underwater imaging system. The light received by camera may include light that has been reflected by an object, and it may include light received without reflection from the object: the latter is called backscatter. Light reflected by the object that reaches the camera has two components: light that has not been scattered in the intervening water, and light that has been scattered at a small angle, called the forward scattered component. Forward scattering generally leads to blur of the image features on the other hand, backscattering generally limits the contrast of the images [3, 4].

Many researchers have developed preprocessing techniques for underwater images using image enhancement methods. Bazeille et al [5] propose an algorithm to pre-process underwater images; the algorithm is automatic and requires no parameter adjustment. Iqbal $\mathrm{K}$. et al [6] using histogram equalization on underwater images to balance the luminance distributions of color. Chambah et al [7] proposed a color correction method based on the automatic color equalization (ACE) model. ACE is perceptual approach inspired by some adaptation mechanisms of the human vision system.

\section{2- PROPERTIES OF WATER AND QUALITY OF LIGHT}

Water is approximately 800 times denser than air, and this density absorbs light quickly .light levels quickly drops as get deeper in water. This leads to decreases contrast and image sharpness. The underwater images often get frustrated from the blue/gray hue of their images, a direct result of the properties of water and the effect of light absorption. This is actually a very common problem and there are several approaches to reintroducing color and clarity to the images [1, $4,8]$.

The refractive index of water is different than air, which makes judging distances difficult the objects view in the image can appear larger than they really are, due to the refractive index difference.

Underwater, light is affected by: Subject distance and weather, Depth, and Surface conditions. Specific frequencies of ambit light get absorbed at different depths, from the longest wavelength the shortest, red nearly disappears at around 5 meters, followed by orange at 10 meters, yellow at 20 meters, and eventually even blue at 60 meters. Due to this, color underwater images require a means of compensation to restore the colors and contrast lost from absorption [6], see figure (1).

\section{3- UNDERWATER ENHANCEMENT FRAMEWORK}

Image enhancement is a process of improving the quality of image by improving its features, the problem of image enhancement is gaining increasingly importance .one of the most significant issue is how to improve the quality of the underwater images in order to streamline the image processing analysis.

\section{4- FUZZY LOGIC}

An idea to solve the problem of underwater images in fuzzy logic manner was the motivation of this work. The Fuzzy logic is relatively young theory (suggested by prof. Zadeh in 1965), the area of applications are very wide: process control, management and decision making, operations research, economic and, pattern recognition and classification. [10] a Fuzzy set is asset whose elements have degrees of membership, an element of a Fuzzy set can be full member (100\% membership) or a partial member (between $0 \%$ and $100 \%$ membership). That is, the membership value assigned to an element is no longer restricted to just two values, but can be 0,1 or any value in-between. Mathematical function which 
defines the degree of an elements membership in a Fuzzy set is called membership function, figure (2) [11].



Figure (1): The three components of underwater optical imaging [4]

Image and Fuzzy set can be modeled in a similar way. Fuzzy set in a universe of $\mathrm{x}$ is associated with a membership degree. Similarly, in the normalized image where the image pixels ranging from $\{0,1,2 \ldots 255\}$ are normalized by 255 , the values obtained are in the interval $[0,1]$. Thus it is a mapping from the image to $[0,1]$. In this way, the image is considered as a Fuzzy set and thus filters are designed.

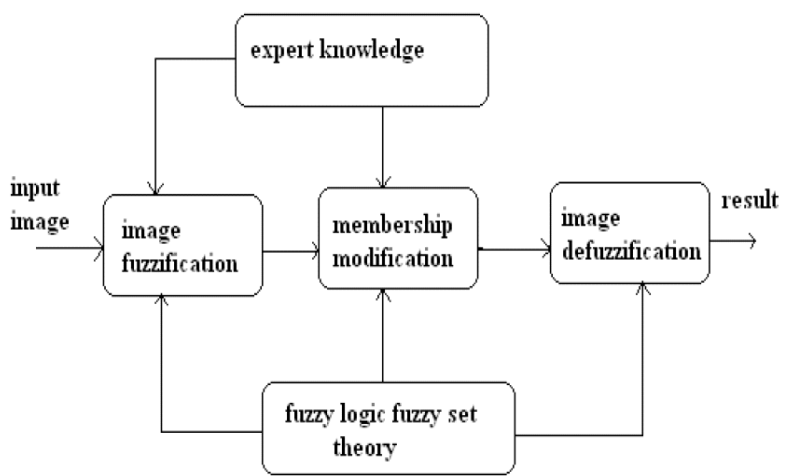

Figure (2): Fuzzy image processing [11]

Fuzzy Histogram Equalization is used to alleviate the effects of edge ringing on the histogram. This is done by using a weighted neighborhood function when computing the histogram. This means that it builds a histogram for each pixel in the image, using a specified number of surrounding pixels. The fuzzy function defined as:

$\mathrm{F}(\mathrm{p})=1 /(1+\beta \mathrm{d}) \quad \ldots(1)$

Where:

$\mathrm{P}$ is the intensity of the pixel being processed $\mathrm{d}$ is the intensity difference between $p$ and a seed point and also $\beta$ controls the opening of the function

The fuzzy histogram equalization (FHE) being proposed is described as [11]:

$I^{\prime}(\mathrm{x}, \mathrm{y})=\operatorname{Ar}(\mathrm{x}, \mathrm{y})+\{\mathrm{I}(\mathrm{x}, \mathrm{y})-\operatorname{Ar}(\mathrm{x}, \mathrm{y})\} \mathrm{F}\{|\mathrm{I}(\mathrm{x}, \mathrm{y})-\operatorname{Ar}(\mathrm{x}, \mathrm{y})|\} \ldots$ (2)

Where:
$\operatorname{Ar}(\mathrm{x}, \mathrm{y})$ denotes the average of the gray levels of the pixels placed radially in distance $r$ away from a seed point.

$\mathrm{F}$ is the fuzzy function

$\mathrm{I}(\mathrm{x}, \mathrm{y})$ is the original intensity of a pixel located at the coordinate $(x, y)$.

$\mathrm{I}^{\prime}(\mathrm{x}, \mathrm{y})$ is the new intensity of the same pixel in the processed

\section{5- IMAGE STATISTICS:}

Useful statistical features of an image are its arithmetic mean, variance and standard deviation. These are well known statistical measures that, when applied to a digit image, can reveal important information.

The arithmetic mean $(\mu)$ is the image's average value. The general equation for calculating the mean of a set of numbers $\mathrm{x}$ would be written as:

$\mu=\frac{\sum_{n, m} x_{i}}{n * m}$

Where $\mathrm{n}, \mathrm{m}$ dimension of image (rows, columns).

The variance $(\sigma)$ is a measure of the distribution or range of pixel values, of an image:

$\sigma=\sqrt{\frac{\sum_{\mathrm{n}, \mathrm{m}}\left(\mathrm{x}_{\mathrm{i}}-\mu\right)^{2}}{\mathrm{n} * \mathrm{~m}}}$

The standard deviation is the square root of the variance. A small standard deviation indicates that the pixel intensities do not stray very far from the mean; a large value indicates a greater range $[9,12]$.

\section{6- ANALYSIS AND RESULTS}

An idea to solve the problem of underwater images using fuzzy histogram equalization was the main motivation of this work. The captured images are diminished due to optical properties of light in an underwater environment. Figure (3) shows the samples of images was taken of an object at different depth under clean water from the search [12].

In this paper we proposed an image enhancement algorithm for underwater image by using FHE system. The proposed approach has different stages: firstly, the contrast stretching of RGB algorithm to equalize the color contrast in the images was used.

The $\mathrm{L} * \mathrm{a} * \mathrm{~b}$ model provides a wider color range by controlling the color elements of the image. Using this technique the RGB color images convert to $\mathrm{L}^{*} \mathrm{a} * \mathrm{~b}$ image to great more range.

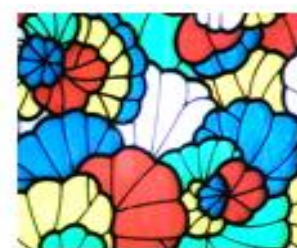

$1 \mathrm{~cm}$

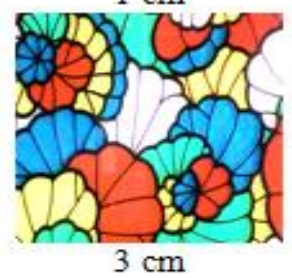

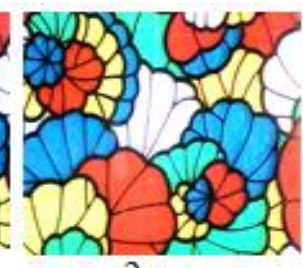

$2 \mathrm{~cm}$






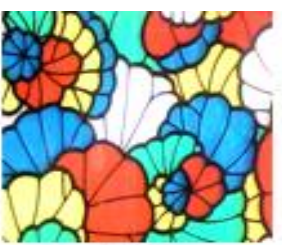

$5 \mathrm{~cm}$

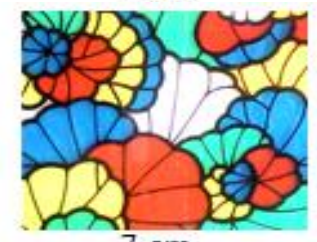

$7 \mathrm{~cm}$

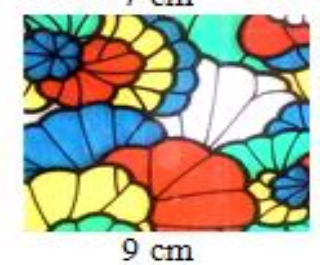

$9 \mathrm{~cm}$
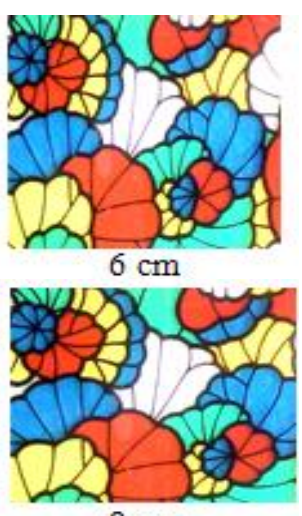

$8 \mathrm{~cm}$

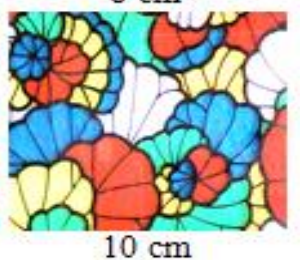

$10 \mathrm{~cm}$

Figure (3): the samples of object images under clean water at different depth.

Then the fuzzy histogram equalization FHE was applied. Figure (4) shows the diagram of the proposed approach. Figure (5) shows the images after histogram equalization processing.

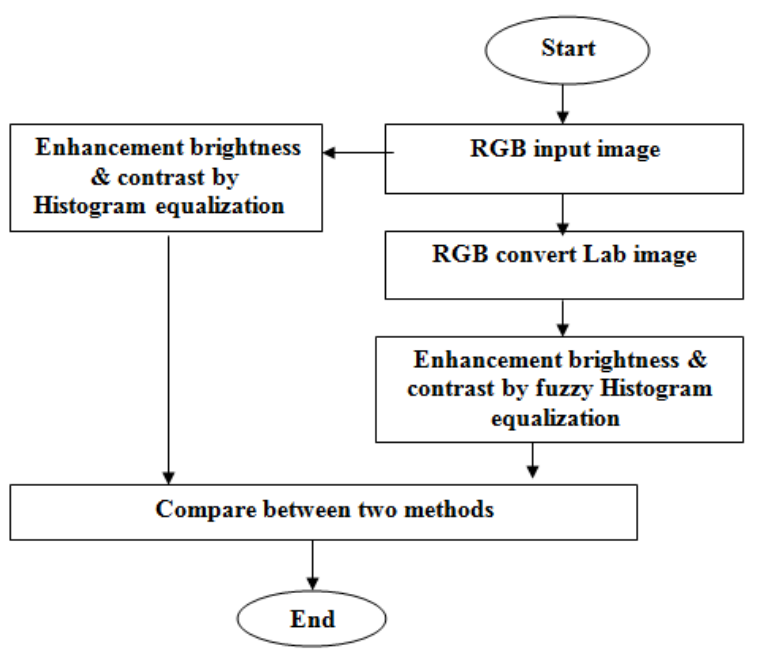

Figure (4): Diagram of FHE technique

Figure (6) shows the images after processing with FHE method proposed in this paper.

A set of statistical measures are computed for every resulting image using both histogram equalization and FHE. Figures (7) and (8) shows mean values for each color components RGBbands for traditional histogram equalization method and the values of other statistic measure respectively.
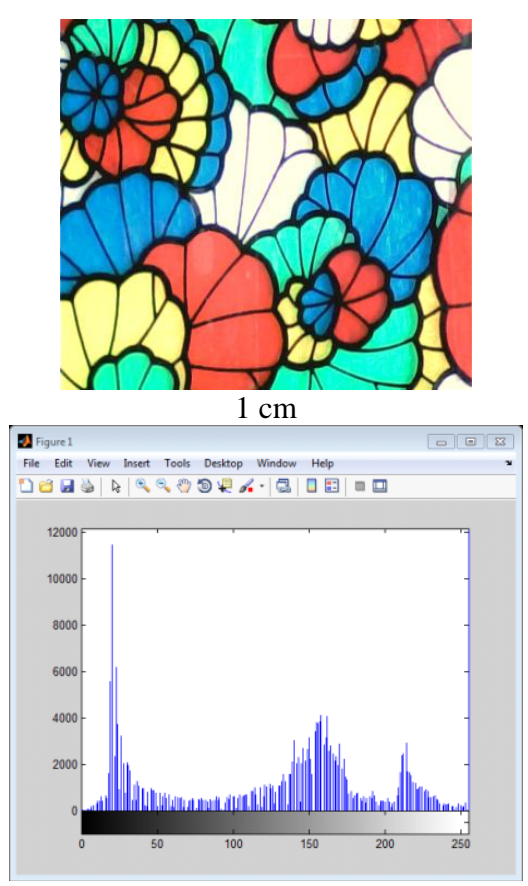

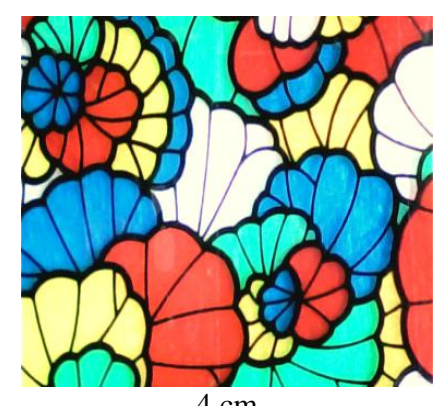

$4 \mathrm{~cm}$

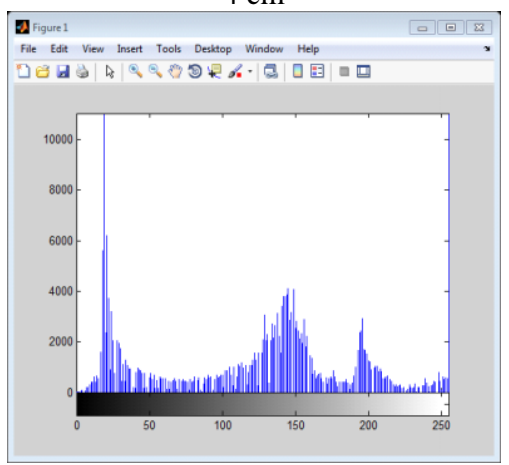

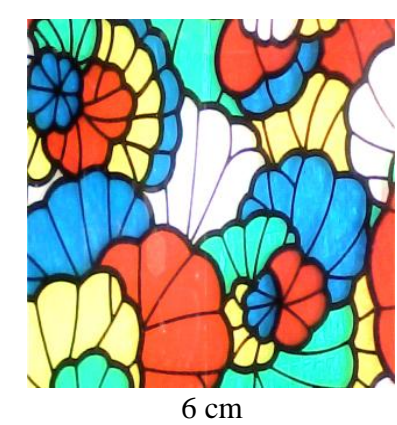

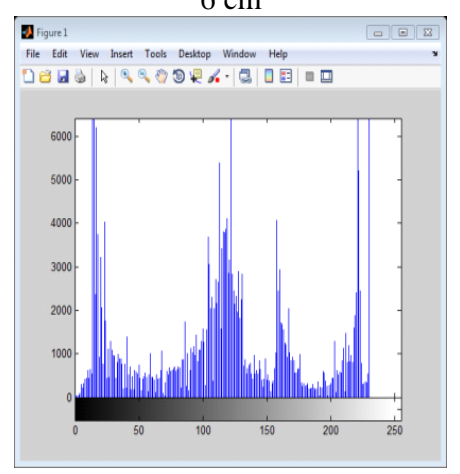




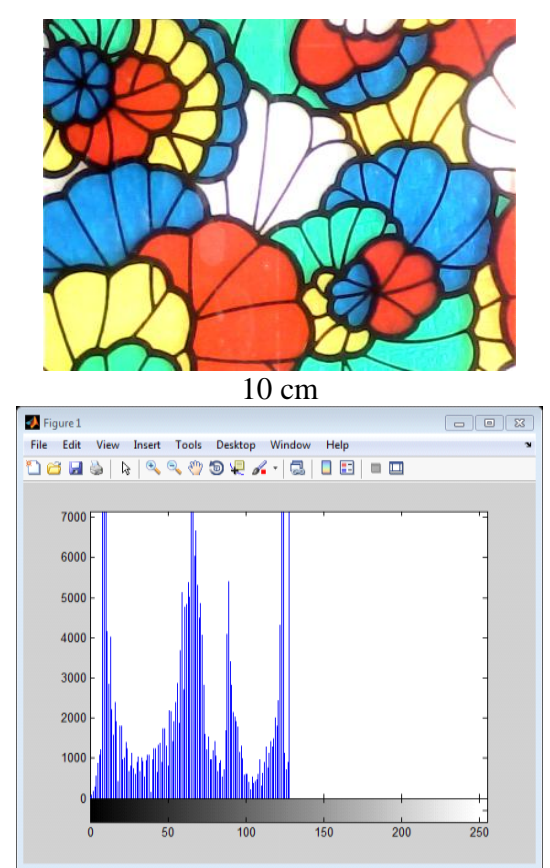

Figure (5): Enhancement brightness \& contrast by Histogram equalization of images
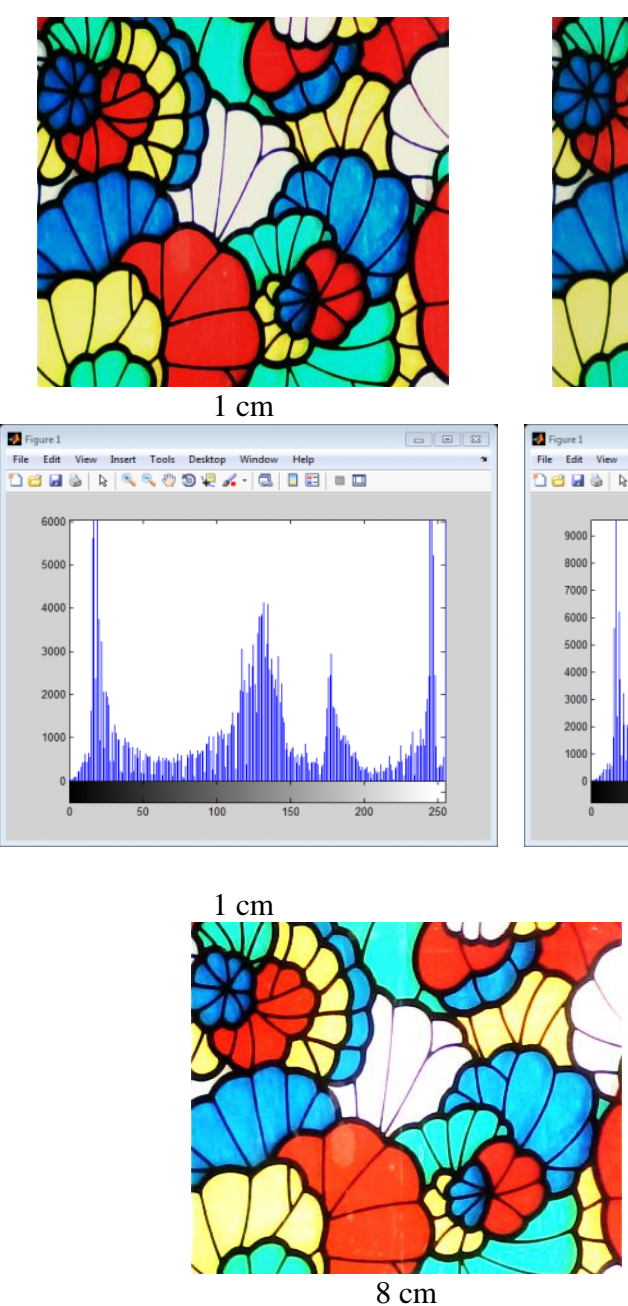

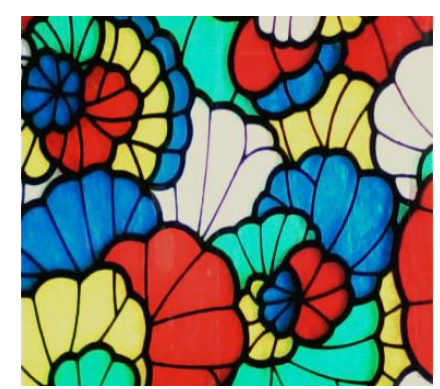

$4 \mathrm{~cm}$
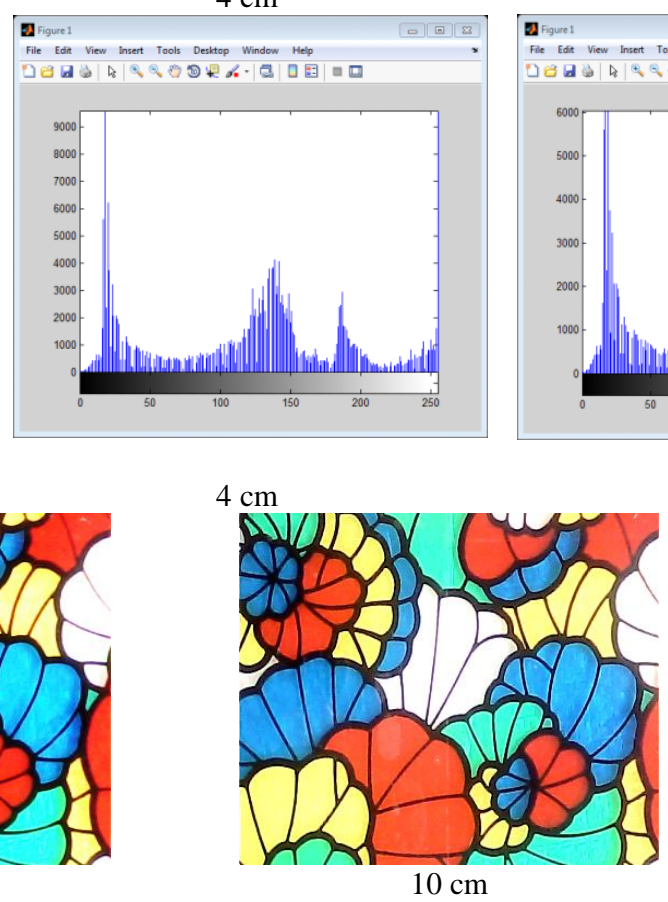
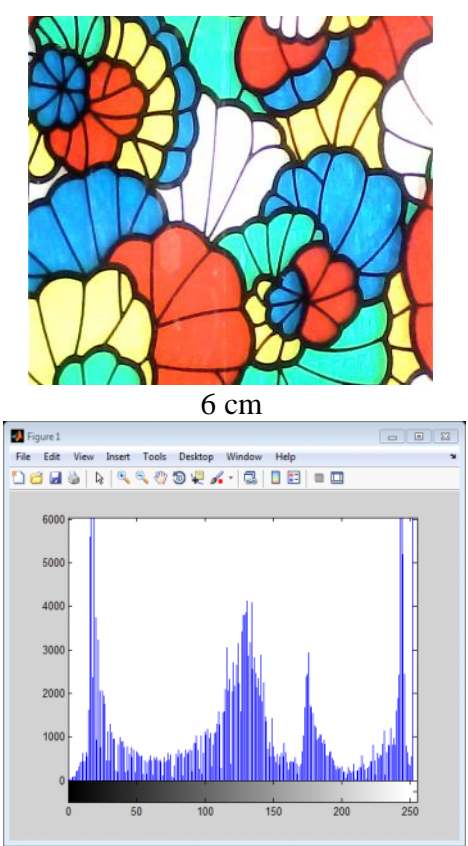

$6 \mathrm{~cm}$ 

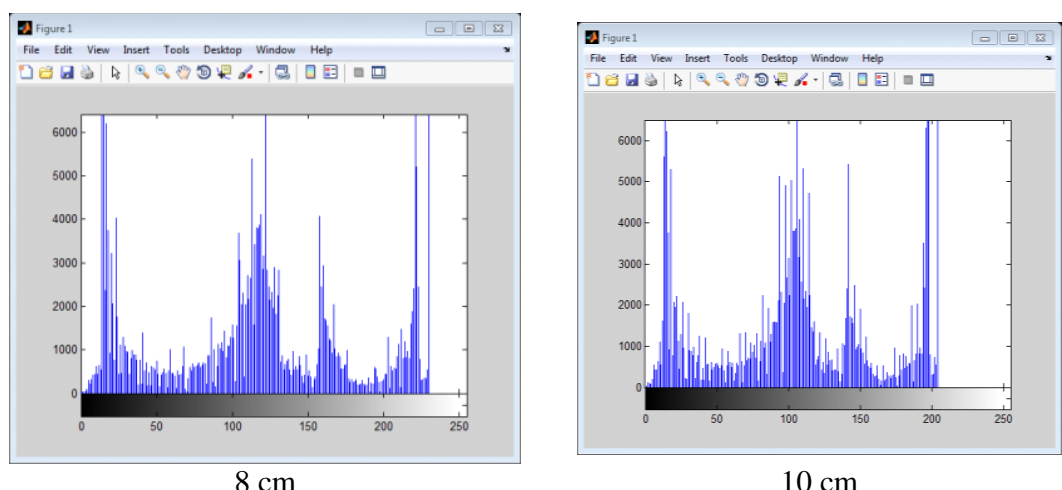

Fig. 6: Enhancement brightness \& contrast by FHE technique

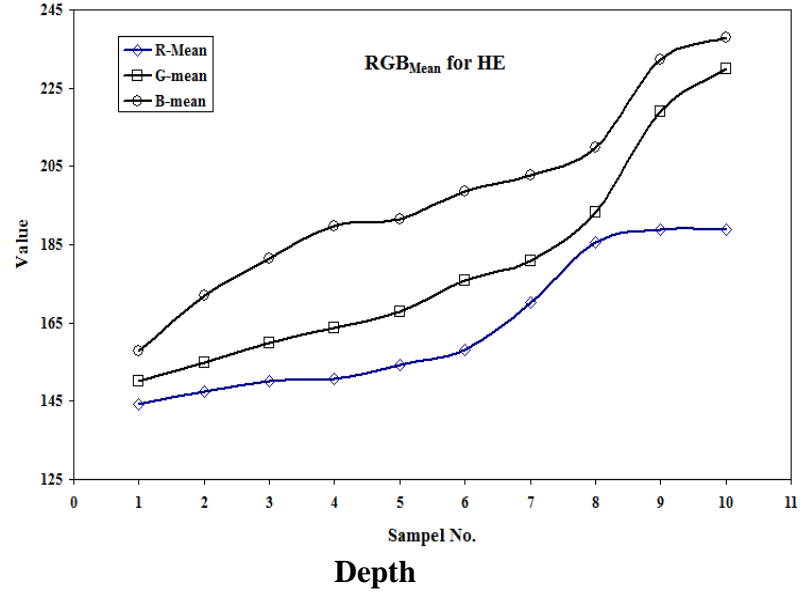

Figure (7) the mean Vs depth of images by HE



Figure (8) Statistic measure Vs depth of images by HE



Depth

Figure (9) the mean Vs depth of images by FHE

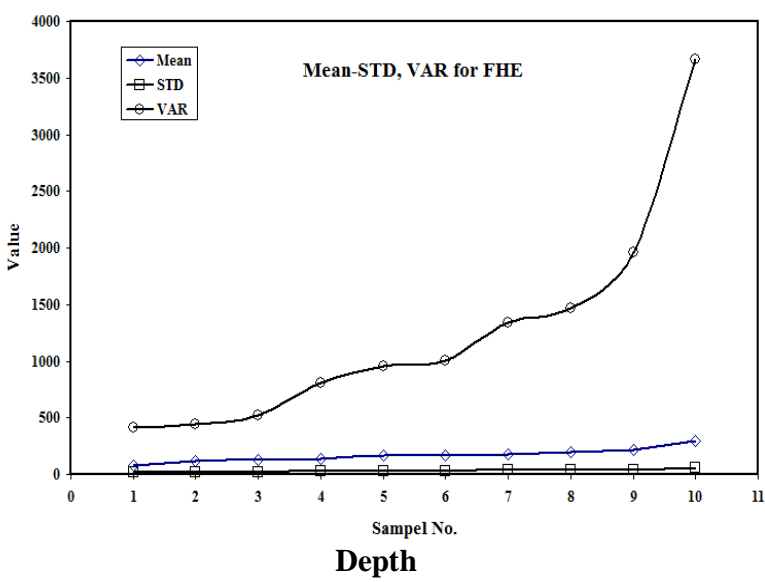

Figure (10) Statistic measure Vs depth of images by FHE

Figures (9) and (10) shows the mean values for each color components RGB-bands for FHE method and the values of other statistic measure respectively. 


\section{CONCLUSIONS}

In this paper, we proposed a FHE enhancement method to enhancing the quality of underwater images. The experimental results demonstrate that the FHE method not only give a better equalization but also improves the contrast of image. This can be clearly seen from the histograms of result.

It's observed from the figures improved of standard deviation, variance values with FHE method in comparison with HE method.

\section{REFERENCES}

[1] John Y. Chiang, Ying Ching Chen and Yung Fu Chen: Underwater image enhancement using WCID. SprigerVerlog Berlin Heidelberg. pp. 373-383 (2011).

[2] Jules S. Jaffe: underwater optical imaging. J. Opt. Soc. Am. 59. pp. 40-42 (1988).

[3] G. Yang, F. Peng, K. Zhao: A Dual-band underwater image denoising and enhancement algorithm. The 2nd International Conference on computer application and system modeling, pp. 1219-1321 (2012).

[4] R. Shettini, S. Corchs: Underwater image processing: state of threat of restoration and image enhancement methods. J. advances in signal processing, 11, pp. 11551168 (2010).

[5] S. Bazeille, I. Quidu, L. Jaulin and J. Malkasse: Proceedings of the European Conference Propagation and Systems, Brest, France (2006).
[6] Iqbal K., Abdul Salam, R., Osman, A.: Underwater image enhancement using an integrated color model. J. Computer Science 34, pp. 2-12 (2007).

[7] Chambah M., Semani D. and Rizzi A.: in color imaging processing, Handcopy and Applications, vol. 5293, pp. 157-168 (2004).

[8] R. Pramunendar, G. Shidik, C. Supriyanto, P. Nurtanti and M. Hariadi: Auto level color correction for underwater image matching optimization, IJCSNS, vol. 13, no. 1, pp. 18-23 (2013).

[9] H. B. Kekre, K. Patil: Standard deviation of mean and variance of rows and columns of images for cbir., world academy of science, engineering and technology 27, , pp. 609-612 (2009).

[10] A. K. John, O. O. Williams: Employing Fuzzy histogram equalization to compute illumination invariance in face recognition system, I. J. intelligent System and application, pp. 54-60 (2012).

[11] K. Venkateshwarlu: Image enhancement using Fuzzy inference system, thesis submitted for the award of degree of master of engineering in computer science and engineering, Thapar University (2010).

[12] A. A. Dawood, G. Karam, A. Majead: Statistical analysis for underwater images, pp. 71-75 (2011). 